\title{
Evaluación de la calidad superficial de piezas mecanizadas a partir del análisis de la fuerza de corte
}

\author{
M. Sánchez-Carrilero ${ }^{(*)}, M$. Marcos $^{(*)}, M$. Álvarez-Alcón ${ }^{(*)}$ y V.M. Sánchez-Corbacho ${ }^{(*)}$
}

Resumen El grado de acabado superficial de una pieza mecanizada es uno de los factores determinantes a la hora de establecer los parámetros de corte necesarios para llevar a cabo el proceso de mecanizado. Generalmente, la evaluación de ese grado de acabado requiere la aplicación de técnicas metrológicas que conllevan paradas de máquina y, a menudo, extracciones de piezas. Este hecho provoca la aparición de una serie de efectos negativos, no sólo en lo que a pérdidas económicas se refiere, sino a fiabilidad en la reproducción de los ensayos. En el presente trabajo se ha estudiado la relación existente entre la fuerza de corte y la rugosidad superficial para una aleación Al-Cu. A partir de esta relación se puede evaluar la calidad superficial de las muestras mecanizadas mediante la determinación de las fuerzas de corte durante el proceso.

Palabras clave: Calidad superficial. Mecanizado. Rugosidad. Fuerzas de corte.

\section{Cutting force analysis as a tool for evaluating the surface quality of machined parts}

\begin{abstract}
Surface quality is one of the most important criteria for establishing the optima cutting parameters needed to perform a machining process. Usually, the evaluation of the degree of the surface finishing requires to apply metrological techniques which involve times out in the production. As a consequence of this, a loss in both the economy of the process and the fiability of the tests reproducibility can be produced. In this work a relation between cutting force and surface roughness has been studied for the turning process of an $\mathrm{Al}-\mathrm{Cu}$ alloy. From this relation, the surface quality of the machined samples can be evaluated by using the cutting forces values acquired during the cutting process.
\end{abstract}

Keywords: Surface quality. Machining. Roughness. Cutting forces.

\section{INTRODUCCIÓN}

En general, el establecimiento de las condiciones óptimas en las que se efectúa un proceso de fabricación está sujeto a un elevado número de parámetros. Incluso, la selección del criterio que determina el grado de optimización presenta una gran dependencia con respecto a las necesidades planteadas y a la infraestructura disponible.

En el caso de procesos de mecanizado de piezas que requieren una alta precisión, el grado de acabado superficial de las mismas es uno de los factores

(*) Dpto. de Ingeniería Mecánica y Diseño Industrial. Univ. de Cádiz. E.S. de Ingeniería. Chile s/n, 11003-Cádiz (España). Tel. (956) 22.17.66. Fax: (956) 22.43.59. e-mail: msc@cain.uca.es determinantes a la hora de establecer los parámetros de corte necesarios para llevar a cabo dichos procesos con resultados óptimos (1). No obstante, generalmente, la evaluación de ese grado de acabado requiere la aplicación de una serie de técnicas metrológicas que suelen llevar consigo paradas de máquina y, a menudo, extracciones de piezas. Este hecho provoca la aparición de una serie de efectos negativos, no sólo en lo que a pérdidas económicas se refiere, sino a fiabilidad en la reproducción de los ensayos. Esto supone la necesidad de buscar una forma de determinación indirecta de la calidad superficial de las piezas durante el proceso de mecanizado. Para ello, es necesario poder establecer relaciones entre parámetros directamente asociados con el grado de acabado superficial y algún 
parámetro de salida cuya medida pueda efectuarse durante el proceso de mecanizado.

En el presente trabajo se ha estudiado la relación existente entre la fuerza de corte y la rugosidad superficial, evaluada a partir de $R_{\mathrm{a}}$ (2) para procesos de cilindrado de una aleación Al-Cu. A partir del establecimiento de esta relación es posible evaluar indirectamente la calidad superficial de las muestras mecanizadas mediante la determinación de las fuerzas de corte durante el proceso, sin necesidad de efectuar paradas intermedias en el mismo.

\section{MÉTODO EXPERIMENTAL}

En nuestro estudio se han empleado barras cilíndricas de la aleación AA-2024 (Al-Cu) en estado T-351, con diámetro variable entre 60 y $90 \mathrm{~mm}$ y longitud comprendida entre 300 y $400 \mathrm{~mm}$. Su composición nominal, en tanto por ciento en masa, se incluye en la tabla I.

Antes de la realización de los ensayos, las muestras fueron sometidas a un proceso inicial de desbaste, con objeto de eliminar tanto la capa natural formada por su exposición al aire como la procedente de procesos de fabricación previos. A continuación, siguiendo las recomendaciones recogidas en (3) se efectuó un proceso de acabado con objeto de minimizar la producción de vibraciones inducidas por la superficie de la pieza sobre la herramienta.

Se efectuaron ensayos de cilindrado en un torno horizontal EmcoTurn-242 T, equipado con Control Numérico Emcotronic TM02 de 21/2 ejes. El Control Numérico, que se encuentra acoplado al Centro de Torneado, permite fijar parámetros como la profundidad de corte, $p$, y controlar la velocidad de corte, $v$, y el avance, $f$. En los ensayos efectuados en nuestro estudio, se fijó una profundidad de corte de $2 \mathrm{~mm}$. Por otro lado, se emplearon velocidades de corte no superiores a los $200 \mathrm{~m} / \mathrm{min}$ y avances comprendidos entre 0,05 y $0,3 \mathrm{~mm} / \mathrm{rev}$.

Para llevar a cabo este estudio preliminar, se decidió elegir herramientas nuevas en cada ensayo, con objeto de evitar los posibles efectos relacionados directa o indirectamente con mecanismos de desgaste (4). Se emplearon plaquitas de Seco con denominación ISO KCMW 11T3 08 FN M-.

En la figura 1 se muestra un esquema del dispositivo experimental empleado para la medida de las

TABLA I.- Composición nominal de la aleación AA2024 (\% en masa)

\section{TABLE I.-AA2024 nominal composition (\% mass)}

\begin{tabular}{|c|c|c|c|c|c|c|c|c|}
\hline $\mathrm{Cu}$ & $\mathrm{Mg}$ & $\mathrm{Mn}$ & $\mathrm{Si}$ & $\mathrm{Fe}$ & $\mathrm{Zn}$ & $\mathrm{Ti}$ & $\mathrm{Cr}$ & $\mathrm{Al}$ \\
\hline 4.00 & 1.50 & 0.60 & 0.50 & 0.50 & 0.25 & 0.15 & 0.10 & Resto \\
\hline
\end{tabular}

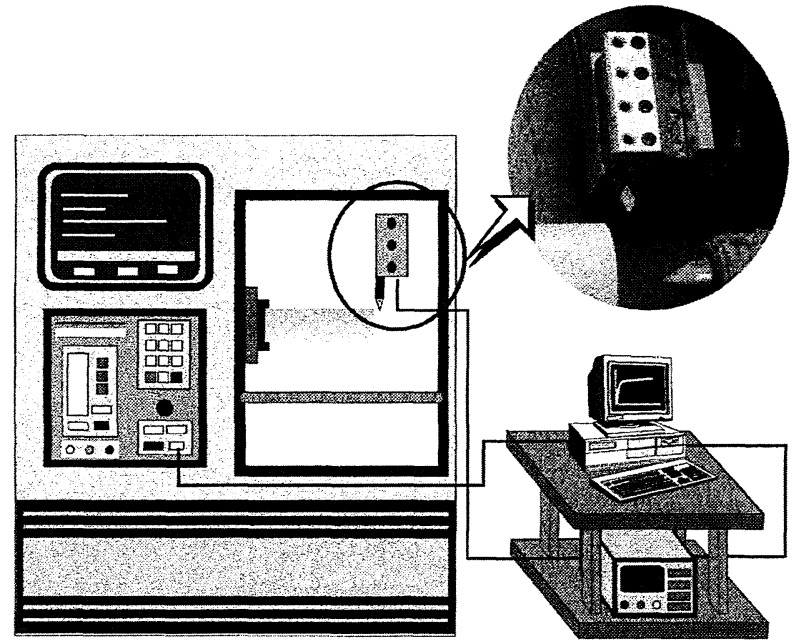

FIG. 1.- Esquema del dispositivo experimental empleado.

FIG. 1.-Experimental device.

fuerzas de corte. Dicho dispositivo consta de un dinamómetro piezoeléctrico KISTLER 9121 de tres componentes, especialmente adaptado al revólver de ocho posiciones del Centro de Torneado CNC, empleándose un portaherramientas 9153A20 para torneado exterior. Los datos dinamométricos fueron adquiridos a través de un amplificador KISTLER Multicharge Channel Amplifier 5019A, de cuatro canales, conectado a un PC, equipado con una tarjeta CIO-AD16 para adquisición de datos. Los valores de la fuerza de corte fueron adquiridos y procesados haciendo uso de la versión 2.0 para DOS del software Cutting forcelturning, de Kistler.

\subsection{Medida de las fuerzas de corte}

Cuando se efectúa un proceso de torneado de una pieza cilíndrica, la fuerza de corte, $F$, puede descomponerse en tres fuerzas ortogonales, como se muestra en la figura 2 , de acuerdo con la ecuación:

$$
F=F_{c}+F_{f}+F_{p}
$$

donde $F_{\mathrm{c}}$ es la fuerza tangencial, $F_{\mathrm{f}}$ es la fuerza según la dirección del avance y $F_{\mathrm{p}}$ es la fuerza radial. El dispositivo dinamométrico empleado permite la medida de las tres componentes ortogonales de la fuerza de corte, la cual se introduce vía una placa superior y se distribuye entre cuatro sensores, capaces de separarlas, que se encuentran ubicados entre dicha placa y la inferior. Cada uno de esos sensores dispone de tres pares de placas toroidales de cuarzo, una sensible a la presión según $Z$, y las otras dos a las tensiones, según $X$ e $Y$, respectivamente (Fig. 3). 


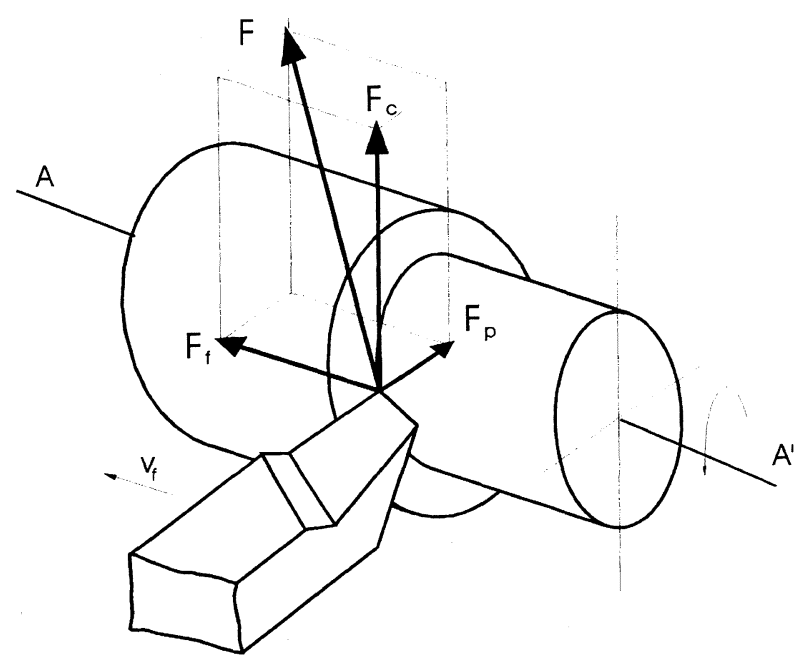

FIG. 2.- Componentes de la fuerza de corte en un proceso de torneado.

FIG. 2.- Cutting force components in a turning process.

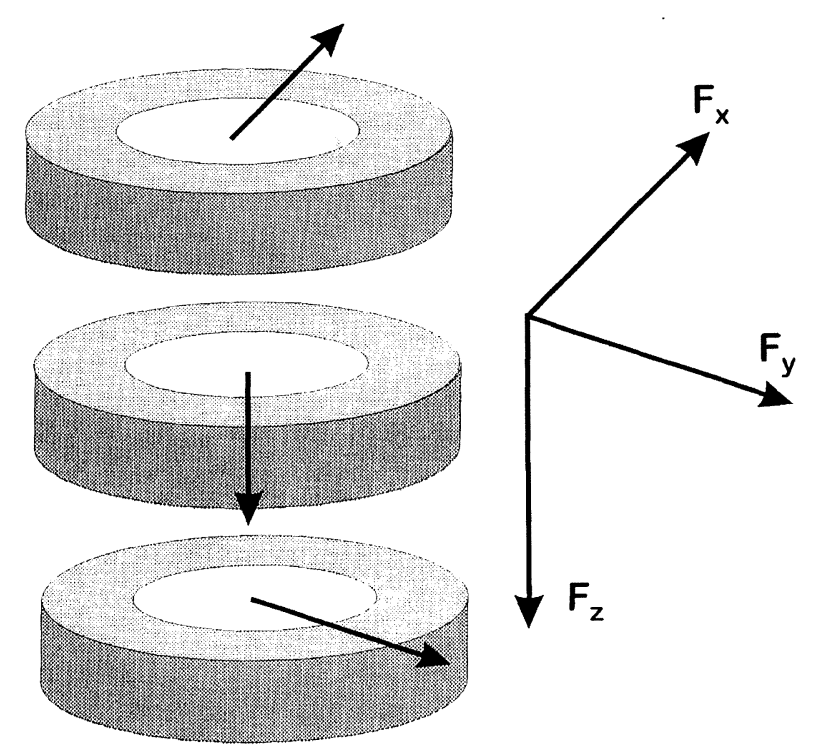

Fig. 3.- Descomposición de la fuerza de entrada en los sensores del dinamómetro.

FIG. 3.- Breaking down of the force introduced by the sensors arranged in the dynamometer.

En general, el valor de $F_{\mathrm{c}}$ es muy superior al de las otras dos componentes. Debido a ello, habitualmente, la fuerza tangencial es considerada como la componente principal de la fuerza de corte $y$, frecuentemente, su valor se asume como el valor de la fuerza de corte (5). Según lo anterior, el estudio incluido en el presente trabajo se ha efectuado a partir del análisis de los valores de $F_{\mathrm{c}}$.

Por otro lado, es necesario resaltar que, en general, los diagramas $F-t$ tienen una forma como la que se muestra en la figura 4 . Como puede observarse

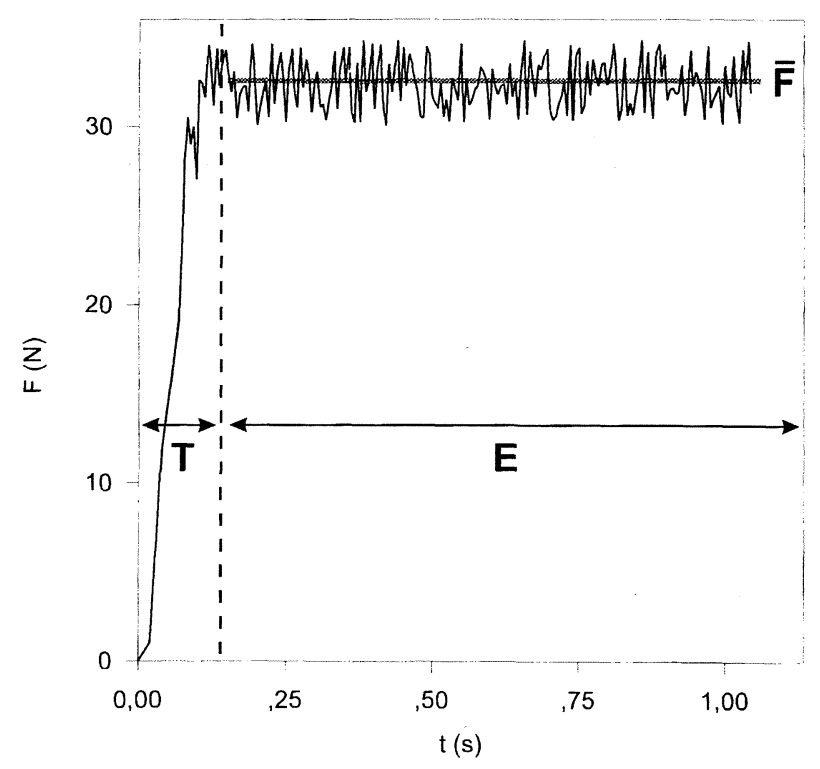

FIG. 4.- Diagrama típico F-t. Obsérvense las zonas correspondientes al estado transitorio $(\mathrm{T})$ y estacionario (E).

FIG. 4.- Typical F-t diagram. Notice the branch corresponding to the transient interval $(T)$ and the zone corresponding to the steady-state $(E)$.

en dicha figura, en estos diagramas se distinguen dos zonas. Una rama inicial, ajustable a una recta de pendiente elevada, correspondiente al estado transitorio, $T$, desde el momento del inicio del corte hasta que el sistema alcanza el estado estacionario, $E$, correspondiente al estado estacionario y ajustable a una recta horizontal. No obstante, y como se aprecia en la figura 4, este estado presenta fluctuaciones aleatorias y/o periódicas de la fuerza alrededor de su valor medio (6 y 7). Si el proceso de corte se encuentra bien diseñado dichas fluctuaciones pueden considerarse como irremediables (7 y 8), por lo que en este trabajo los valores de $F$ considerado, se han evaluado a partir del valor medio en la zona de los diagramas $F$ - $t$ correspondientes al estado estacionario.

\section{RESULTADOS Y DISCUSIÓN}

En la figura 5 se muestra, en escala logarítmica, la evolución de la fuerza de corte, asumida como $F_{\text {c }}$, con la velocidad de corte para distintos avances. Por una parte, puede observarse cómo dicha evolución depende del avance considerado. En efecto, como se aprecia en la figura 5, la fuerza de corte aumenta ligeramente cuando se trabaja con avances inferiores a $0,2 \mathrm{~mm} / \mathrm{rev}$, mientras que muestra una tendencia contraria cuando el proceso de mecanizado se efectúa con avances superiores. Por otro lado, en la misma figura, se puede observar cómo los 


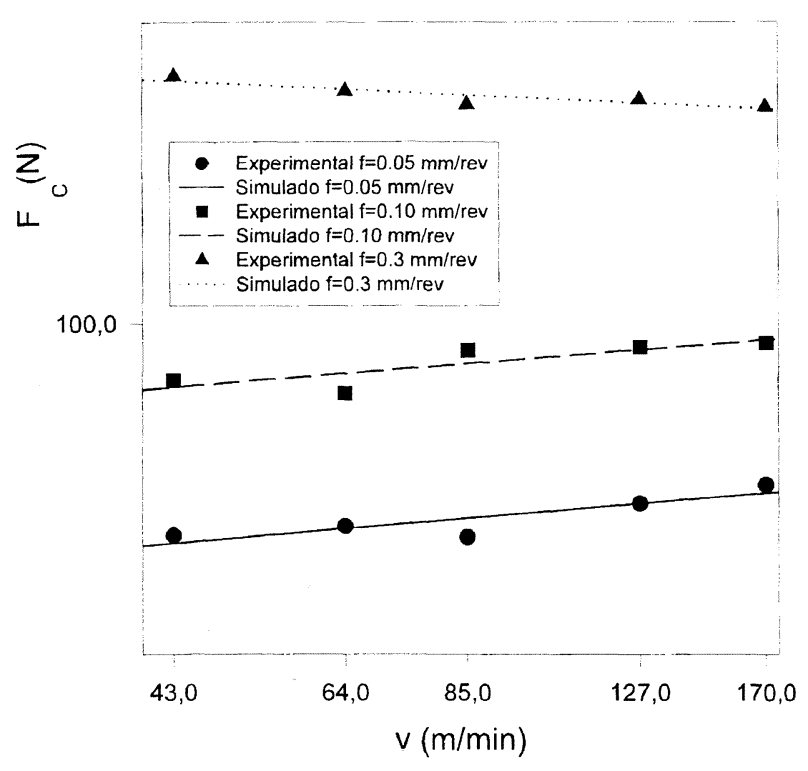

FIG. 5.- Diagramas $\mathrm{F}_{\mathrm{c}}-\mathrm{v}$ para distintos avances.

FIG. 5.- $F_{c}$-v diagrams for different feeds supplied.

puntos muestran una tendencia lineal por lo que puede ensayarse un modelo potencial en la forma:

$$
F_{c} \propto v^{n}
$$

El ajuste de los puntos experimentales al modelo propuesto se ha incluido en el diagrama de la figura 5 .

Una relación potencial del estilo de la recogida en la ec. [2] puede proponerse para la evolución de $F_{\mathrm{c}}$ con el avance $f$ :

$$
F_{c} \propto f^{m}
$$

de acuerdo con lo observado en la figura 6 . En esta figura, se incluye, también, el intervalo de confianza al $95 \%$ para los datos tratados.

A partir de ambas relaciones potenciales puede asumirse un modelo de Taylor para el comportamiento de $F_{\mathrm{c}}$ :

$$
F_{c} \propto v^{n} f^{m}
$$

Como se ha comentado anteriormente, la calidad de acabado superficial de las piezas cilindradas fue evaluada efectuando medidas del parámetro $R_{\mathrm{a}}$ en cuatro puntos de su superficie elegidos al azar. En la figura 7 se muestra la evolución de dicho parámetro con la velocidad de corte para distintos avances seleccionados. Como puede comprobarse, la evolución de $R_{\mathrm{a}}$ presenta tendencias semejantes a las mostradas por $F_{\mathrm{c}}$ con respecto a la velocidad de corte, por lo que puede proponerse un modelo de comportamiento similar al de la ec [2]. De igual

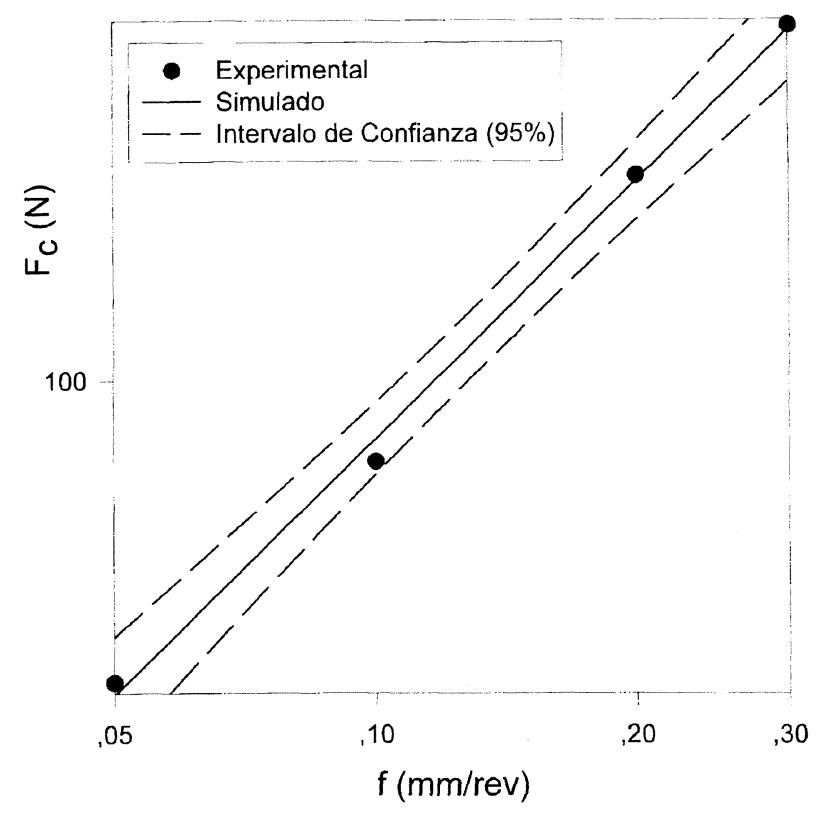

FIG. 6.- Diagrama $F_{c}-$ f. Se incluyen la recta de ajuste y el intervalo de confianza al $95 \%$.

FIG. 6.- $F_{c}-f$ diagram including fitted curve and the confidence interval (95\%).

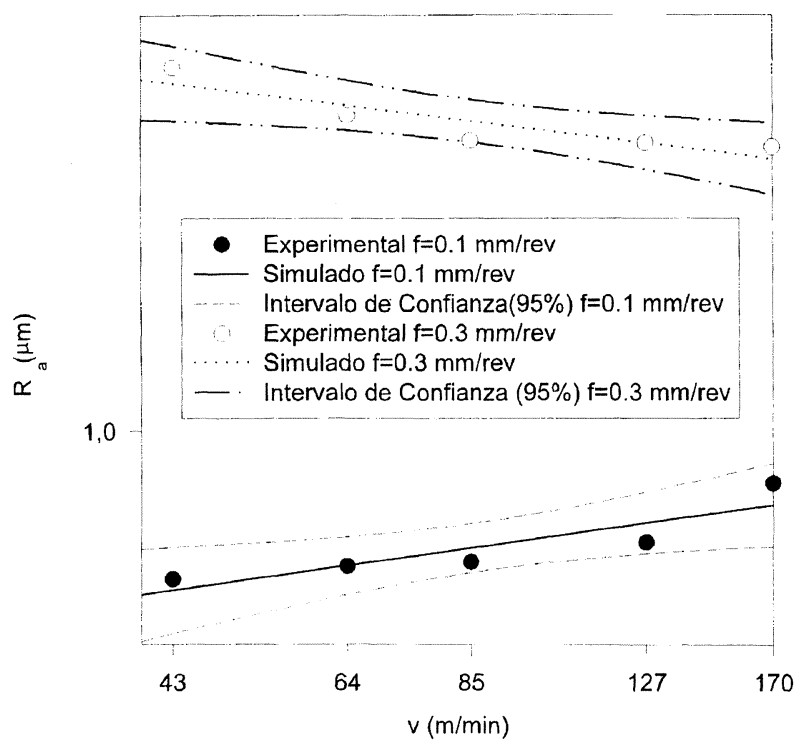

FIG. 7.- Diagramas $\mathrm{R}_{\mathrm{a}}-\mathrm{v}$. Se incluyen las rectas de ajuste y los intervalos de confianza (95\%) correspondientes.

FIG. 7.- $R_{a}-v$ diagrams including fitted curves and confidence intervals (95\%).

forma, en la figura 8 , se representa la evolución de $R_{\mathrm{a}}$ con el avance en las condiciones de corte del diagrama de la figura 6 . La similitud entre ambos diagramas permite proponer un modelo semejante al de la ec. [3] para el comportamiento de $R_{\mathrm{a}}$ frente a este parámetro y, consecuentemente, la evolución de $R_{\mathrm{a}} \operatorname{con} f$ y $v$ estaría gobernada por una ley como 


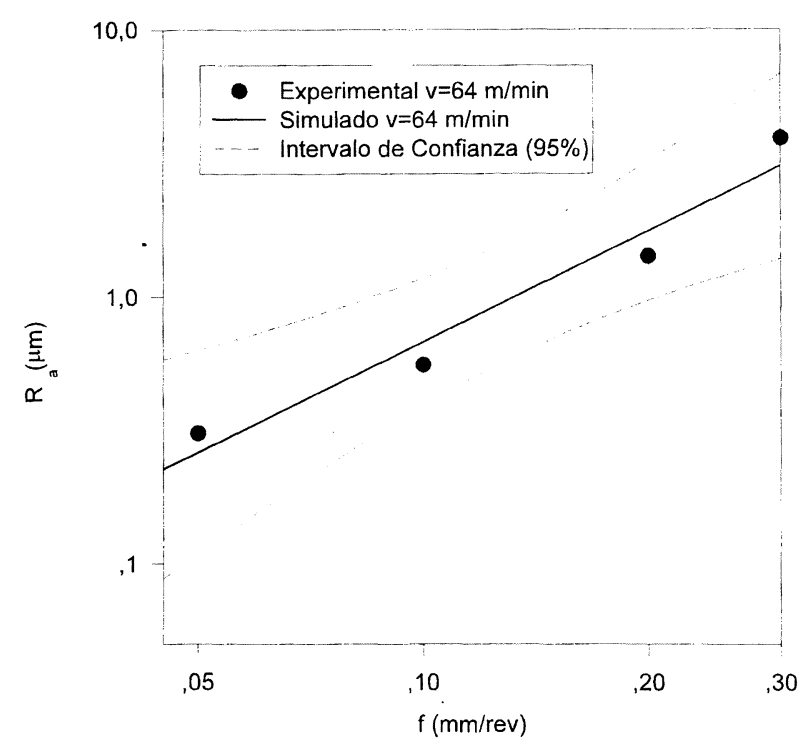

FIG. 8.- Diagrama $R_{a}-f$. Se incluye la recta de ajuste y el intervalo de confianza al $95 \%$.

FIG. 8. $-R_{a}-f$ diagram including fitted curve and the confidence interval (95\%).

la expresada en la ec. [4]. Por tanto, puede pensarse en una relación potencial entre $R_{\mathrm{a}}$ y $F_{\mathrm{c}}$ en la forma:

$$
R_{a}=a F_{c}^{b}
$$

En esta ecuación, el coeficiente $a$ y el exponente $b$ dependen de las condiciones de corte, esto es, el avance y la velocidad de corte. Por otra parte, esta ecuación permite predecir la calidad superficial de las muestras ensayadas a partir de las medidas de los valores de la fuerza de corte.

\section{CONCLUSIONES}

El seguimiento del grado de acabado superficial de piezas mecanizadas requiere la aplicación de téc- nicas metrológicas que conllevan paradas de máquina $\mathrm{y}$, a menudo, extracciones de piezas. Esto conlleva una serie de efectos negativos, tanto en lo que a pérdidas económicas se refiere, como en la fiabilidad en la reproducción de los ensayos.

En el presente trabajo se ha estudiado la evolución con el avance y la velocidad de corte, de la fuerza de corte, medida a través de un dinamómetro piezoeléctrico, y de la calidad superficial, evaluada a través del parámetro $R_{\mathrm{a}}$, en procesos de cilindrado de la aleación AA2024. La comparación del comportamiento de $F_{\mathrm{c}}$ y $R_{\mathrm{a}}$ ha permitido establecer una relación potencial entre ambas. A partir de este modelo matemático es posible efectuar el seguimiento del acabado superficial de las piezas mecanizadas sin necesidad de llevar a cabo paradas de máquina, evitando así, los efectos negativos anteriormente mencionados.

\section{REFERENCIAS}

(1) Boothroyd, G. y Knight, W.A. Fundamentals of Machining and Machine Tools, Marcel Dekker Inc., Nueva York (EE.UU.), 1989.

(2) American National Standards, Surface Texture: Roughness, Waviness and Lay, ANSI-ASME B46-1-1985, Am. Soc. Mech. Engrs. 1985.

(3) Elbestawi, M.A., Ismail, F., Du, R. y Ellagaddi, B.C. Jour. Eng. Ind., 116 (11), 1994: 435.

(4) Young, H.T., Mathew, P. y Oxley, P.L.B. Proc. Inst. Mech. Engrs., 201 (3), 1987: 213.

(5) Sánchez Carrilero, M. y Marcos, M. Relaciones Paramétricas en el Mecanizado, Serv. Pub. UCA, Cádiz (España), 1994.

(6) Andreasen, J.L. y De Chiffre, L. Ann. of the CIRP, 42 (1), 1993: 45.

(7) Sánchez Carrilero, M., Marcos, M., Álvarez, M., SÁnchez LóPez, M. y Contreras, J.P. An. Ing. Mec., 11 (4), 1997: 59.

(8) Sánchez Carrilero, M., Marcos, M. y Sánchez Corbacho, V.M. J. Mech. Beh. Mater., 7 (3), 1996: 167. 